\title{
Design of antenna control system P-Fuzzy-PI controller
}

\author{
Tao Min $^{1}$, Li Shuzhao ${ }^{2}$, Jia Yunxiang ${ }^{1}$, Zhang Guiping ${ }^{1}$ \\ ${ }^{1}$ China Satellite Maritime Tracking and Control Department, Jiangsu, Jiangyin, 214431, China \\ ${ }^{2}$ School of Innovation Experiment, Dalian, Liaoning, 116200, China
}

Keywords: antenna control; robustness; P-Fuzzy-PI

\begin{abstract}
The antenna control system for the problem encountered in the application, starting from the control algorithm to analyze the composition of the antenna control systems, introduces the antenna control system design, and antenna control unit, an antenna drive unit, shaft encoder unit and security unit with a detailed description. Propose proportion - fuzzy - proportional integral (P-Fuzzy-PI) controller, and as a basis for the design of the control algorithm used, and its simulation, simulation results show that reducing the overshoot, improving system dynamic performance, confirming the correctness of the controller.
\end{abstract}

\section{Introduction}

With the rapid development of electronic technology, geostationary satellite system has been widely used, while also seeking to develop domestic and international polar-orbiting satellite system, so satellite remote sensing technology services to scientific experiments, earth resources exploration, environmental monitoring, marine and meteorological information and disaster detection and other services. In the ground station equipment, for remote sensing, monitoring and control goniometer, the antenna control systems are an important part.

Analysis, antenna control system classic PID (proportional, integral, derivative) controller is slow and there is a corresponding large overshoot shortcomings, in order to improve the performance of the system, proposed and studied the P-Fuzzy-PI controller.

\section{Antenna Control System Analysis and Design}

Antenna control system consists of an antenna control unit, consisting of an antenna drive unit, shaft encoder unit and a security unit, etc., the system block diagram shown in Figure 1.

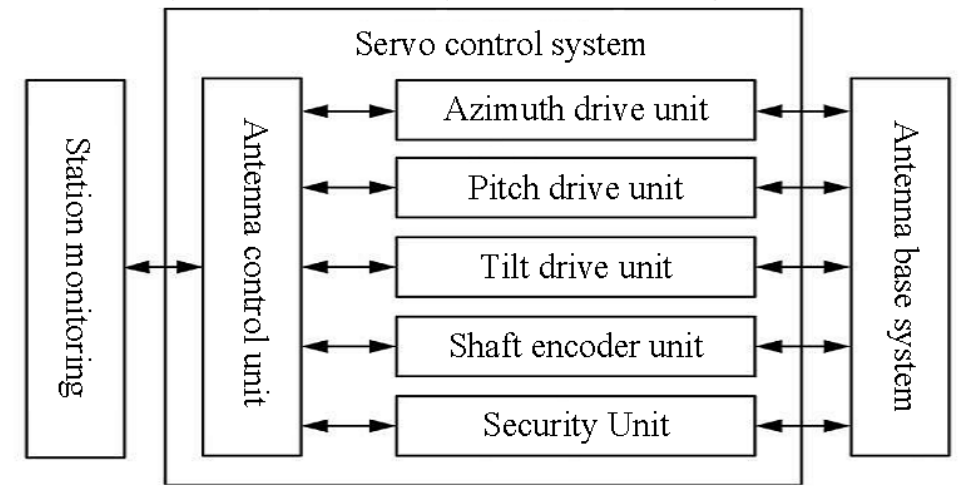

Figure 1. The antenna control system

\subsection{Antenna control unit}

Antenna control unit (ACU) by the data acquisition card, D / A (digital / analog) converter card, network card, general industrial control computer and design their own circuit boards, antenna control software installed in the ACU.

ACU is a core part of the tracking control system, mainly to complete the task of tracking the automatic monitoring, data processing, coordination and control devices within the system to complete the target precision tracking, complete motion attitude control antennas and real-time computing and implementation of control strategies, At the same time recording information for 
each measurement, analysis and display, management subsystem to report to the station control equipment status information and antenna angle.

\subsection{Antenna drive unit}

Antenna azimuth axis drive unit into the drive unit, pitch-axis drive three partial units and tilt drive unit, are driven antenna azimuth axis, pitch axis and tilt axis. In the high-precision antenna system, stability and other indicators backlash positioning accuracy of the system and the system has a great influence, and therefore generally different backlash measures to ensure that the system of indicators, is a dual-motor backlash good way.

The basic principle of double motor backlash is to use two identical motors drive the same load through respective deceleration device, moment by moment bias and equalization circuitry to control the working status of the motor when the load torque is small, two motor output the opposite direction of the moment. A motor working condition for drive motor, the status of another motor passive brake state, two states with the change of direction of the load torque shift, and this change is smooth and continuous, thus ensuring load low-speed operation is smooth and no backlash occurs. With the increase of the passive state of the motor load torque also becomes active state, two motor drag load together.

\subsection{Shaft encoder unit}

Rotary encoder unit mainly to the amount of angular position of the antenna to digital conversion, using a rotary transformer, angle Converter (RDC) combining circuit program, the advantage of this program is high conversion accuracy, digital signal directly, easy to use, and less susceptible to interference, the accuracy of angle selection according to actual requirements.

Test resolver using precision-made sleeve shaft 1:32 on pole dual-channel rotary transformer, RDC using 14 encoders, through the combination of dual-channel Coarse get 17 precision data.

\subsection{Security Unit}

Security unit by the locking mechanism and limit switch composed of an antenna mounted on the respective base position. After collection of the need for the antenna lock operation to ensure in windy weather antenna is not damaged. Limit switches are used to limit the range of rotation of the antenna, the antenna to a limit switch, the limit switch issue limit signals, prohibit the antenna rotation to ensure the safety of the antenna.

\section{Design P-Fuzzy-PI controller}

To improve the accuracy and tracking performance fuzzy controller based, it is necessary to take more languages to linguistic variables value, and sub-file more detailed the better performance, but also greatly increase the amount of computing brings, as well as the fuzzy control rule table it is difficult to grasp, debugging more difficult, or does not meet the requirements of real-time control, a method to resolve this contradiction in theory within segments with different control methods to achieve control. When the deviation is greater than the threshold with a proportional controller to increase the response speed of the system, speed up the response process, switching into the fuzzy control when the deviation is less than the threshold, in order to improve the damping performance of the system, reducing the overshoot response process. This combines the advantages of proportional control and fuzzy control. In this method, the universe fuzzy control is only part of the whole universe, it is the equivalent of fuzzy control theory is compressed domain, equivalent to linguistic variables and sub-file number increases, improved sensitivity and control accuracy.

However, since no integral part of fuzzy control, the input control is a discrete and limited, and the control surfaces are smooth rather than ladder-type, and thus ultimately there must be more than the difference between the steady-state, and the emergence of small amplitude oscillation phenomenon in the vicinity of the equilibrium point. The PI control in the vicinity of the equilibrium point of a small range of adjustment effect is desirable, its integral role could eventually eliminate residual error. Structure P-Fuzzy-PI controller is shown in Figure 2. 


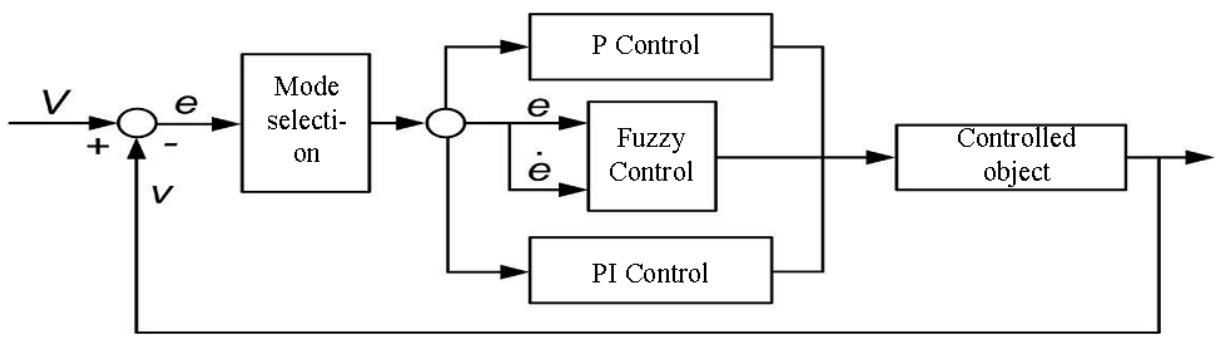

Figure 2. P-Fuzzy-PI controller structure

System control voltage $\mathrm{V}$ and $\mathrm{A} / \mathrm{D}$ conversion values a linear relationship,

$$
V=5+\frac{4096^{*} a}{20}
$$

The value of $\mathrm{V}$ as the control amount to discuss, in FIG. $2 \mathrm{~V}$ is the set value, the figure can be obtained from:

$$
e=R-r, \bar{e}=\frac{d e}{d t}
$$

Effect fuzzy controller is adjusted in accordance with both the value of $\mathrm{V}$, thereby controlling the rotation of the antenna, the system $\mathrm{V}$ range $[-5,5]$.

\subsection{P control}

P controller algorithm formula is:

$$
u=k p^{*} e \quad\left(e \geq 5^{\circ} \text { or } e \leq 5^{\circ}\right)
$$

In the above formula, $\mathrm{kp}=0.8$.

When the absolute value of the deviation is greater than 5 degrees with a proportional controller, proportional controller was characterized by a fast response, we can accelerate the process of the system response.

\subsection{PI control}

Open-loop PI controller transfer function is:

$$
K(s)=\frac{K_{a}(\tau s+1)}{s^{2}\left(T_{s}^{22}+2 \xi T_{s}+1\right)}
$$

Usually in discrete antenna control system incremental digital PI control algorithm, the so-called incremental PI refers to the digital output of the regulator is to control the amount of the increment of $U(\mathrm{k})$, which is expressed as:

$$
U(k)=K_{p}^{*}\left[e(k)-e(k-1)+e(k) * T_{s} / T_{i}\right]
$$

PI algorithm poor second-order non-static system, it can eliminate the static error of the system, improve the tracking accuracy of the system.

\subsection{Fuzzy Control}

Membership functions shown in Figure 3. 


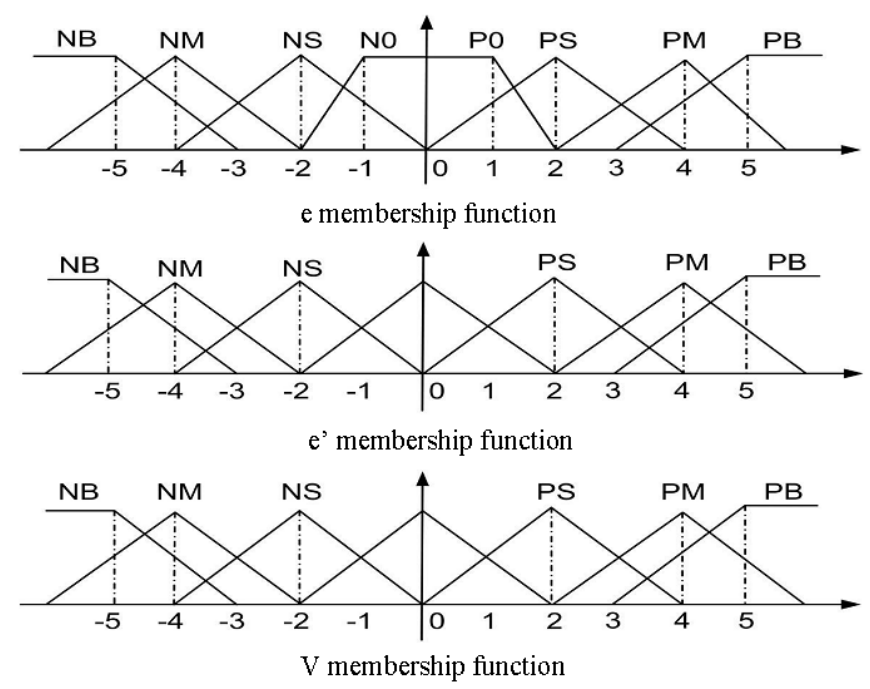

Figure 3. Membership function diagram

In Fig. 3, when $-5 \leq \mathrm{e} \leq-1$ or $1 \leq \mathrm{e} \leq 5$ using Fuzzy control, antenna control system design using look-up table fuzzy controller. Since the hardware configuration of the fuzzy controller IPC to achieve, so in order to save memory and run time, the actual control process is not performed in real time reasoning, but offline calculate the corresponding relationship between the input output and stored in the form of a table IPC memory, IPC inquiry form based on the measured input value, the corresponding output value.

\section{Simulation results analysis}

Matlab simulation environment based on system simulation to get the system response curve shown in Figure 4.

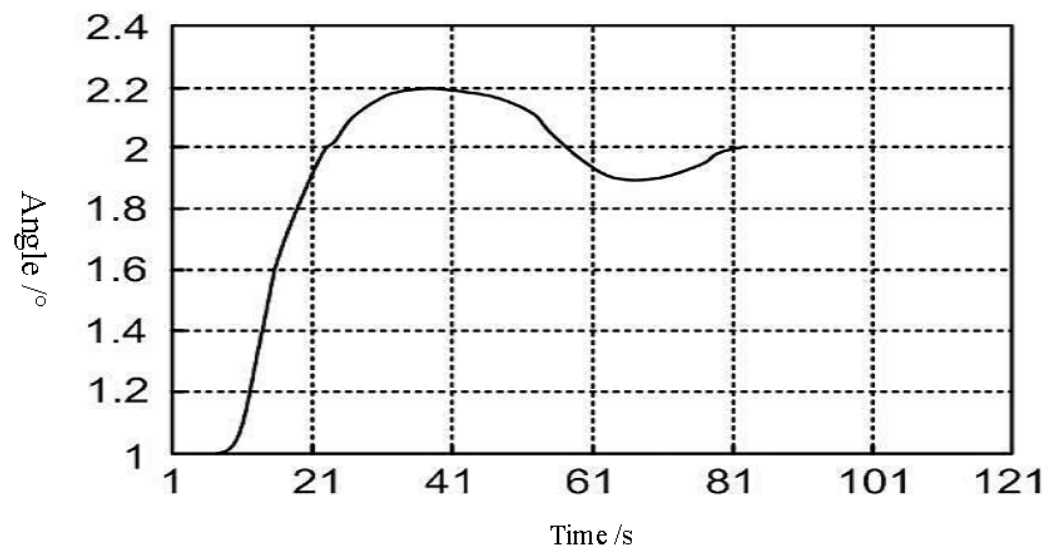

Figure 4. System response curve

Graphics can be drawn from the rise time of the system is $0.5 \mathrm{~s}$, the shock number 1 times. The maximum overshoot of 0.2 degrees, the static error is 0 , P-Fuzzy-PI controller is compared to the conventional PID controller, greatly improving the adaptability of the system and against external interference and internal parameter variation robustness, reduced overshoot, improved dynamic performance. Compared with the simple fuzzy controller, he reduced the steady-state error and improve the stability of the equilibrium point, verify the correctness of P-Fuzzy-PI controller.

\section{Conclusion}

P-Fuzzy-PI controller for antenna control system designed to solve the antenna control system super tune is too large, the shortcomings of slow response time, the whole system has real-time, the system of adjustable parameters, high reliability advantages, the tracking system can achieve accuracy to meet the technical requirements of the project. 


\section{References}

[1] Sun Debao. automatic control theory [M] Beijing: Chemical Industry Press, 2012: 100-110.

[2] Wei Yingjie. Weather radar target fed before the ship station [J]. Radio Engineering, 2007, 37 (5): 44-46.

[3] Hanwen Ze, Dong Xufeng. Optimal tracking of satellite earth station antenna [J] Radio Engineering, 2013, 35 (5): 44-46.

[4] Zhu Jing. Fuzzy control theory and application [M] Beijing: Mechanical Industry Press, 2007: 150-170. 